\title{
Intervention bimanual stimulates the abilities in infants with asymmetry
}

\author{
A intervenção bimanual \\ estimula as habilidades em \\ bebês com assimetria
}

Mayara Thais Correr Ribeiro (1)

Luzia lara Pfeifer (D)*

Universidade de São Paulo (USP), Ribeirão Preto, SP, Brazil

Date of first submission: October 20, 2020

Last received: February 14, 2021

Accepted: March 16, 2021

Associate editor: Clynton Lourenço Correa

* Correspondence: luziara@fmrp.usp.br

\section{Abstract}

Introduction: The cerebral palsy and brachial plexus injury may cause asymmetry in the use of the upper limbs (UL). This condition requires early treatment to reduce the impact of the child's life; therefore, several proposed interventions aim to increase their functional independence. The Constraint-Induced Movement Therapy (CIMT) and Hand-Arm Bimanual Intensive Therapy (HABIT) have been widely considered effective interventions to improve hand function. Objective: Investigate the effects of an intervention protocol based on the CIMT and HABIT theoretical foundations in the stimulation of manual function in infants with the UL asymmetry. Methods: Five infants (6-24 months) participated in the study. To evaluate the motor function of infants we used Pediatric Motor Activity Log (PMAL-R) and Manual Function Evaluation (AMIGO), and to assess the caregiver's perception of the participation of the infant in daily tasks, we used the Pediatric Disability Assessment Inventory (PEDI). All evaluations occurred before, immediately after the intervention, and after four months for follow-up recording, and were analyzed descriptively by Jacobson- Truax method. Results: The results between evaluation and reevaluation demonstrated evolution in all aspects studied. In the PEDI self-care session, an average of 38.6 ( \pm 8.4$)$ - $44.2( \pm 7.4)$; PEDI Mobility: $28.8( \pm 20.3)$ 36.28 ( \pm 21.7$)$; PEDI Social Function: $40.1( \pm 10.2)-42.3$ ( \pm 8.9 ). The PMAL-R quantity and quality results evidence a highly positive clinical significance in all infants. Conclusion: The application of the modified restriction intervention protocol resulted in reliable and clinically significant changes in all cases.

Keywords: Infants. Clinical efficacy. Upper extremit. 


\section{Resumo}

Introdução: A paralisia cerebral e a lesão do plexo braquial podem causar assimetria no uso dos membros superiores (MS). Esta condição requer tratamento precoce para reduzir o impacto na vida do indivíduo, portanto várias intervenções têm sido propostas com o objetivo final de aumentar sua independência funcional. A terapia de movimento induzido por restrição (CIMT) e a terapia intensiva bimanual de mãobraço (HABIT) têm sido amplamente eficazes para aumentar a função da mão. Objetivo: Investigar os efeitos de um protocolo de intervenção beseado nos fundamentos teóricos da CIMT e do HABIT, na estimulação da função manual em bebês com assimetria de MS. Métodos: Cinco crianças (6-24 meses) participaram do estudo. Para avaliar a função motora dos lactentes foram utilizados o Pediatric Motor Activity Log (PMAL) e a Avaliação de Função Manual (AMIGO); o Pediatric Disability Assessment Inventory (PEDI) foi aplicado aos cuidadores, a fim de avaliar sua percepção da participação funcional do lactente nas tarefas diárias. Todas as avaliações ocorreram antes, imediatamente após a intervenção e após quatro meses para registro de acompanhamento. Os dados foram analisados descritivamente pelo método de JacobsonTruax. Resultados: Os resultados entre avaliação e reavaliação demonstraram evolução em todos os aspectos estudados. $\mathrm{Na}$ sessão de autocuidado do PEDI, a média de 38,6 $( \pm 8,4)$ foi para 44,2 $( \pm 7,4) ; P E D I$ - Mobilidade: de $28,8( \pm 20,3)$ a 36,28 $( \pm 21,7) ;$ PEDI - Função Social: de 40,1 $( \pm 10,2)$ a 42,3 ( $\pm 8,9)$. Os resultados de quantidade e qualidade do PMAL-R evidenciam um significado clínico altamente positivo em todos os bebês.

Conclusão: $A$ aplicação do protocolo de intervenção de restrição modificado resultou em alterações confiáveis e clinicamente significativas em todos os casos.

Palavras-chave: Bebês. Eficácia clínica. Extremidade superior.

\section{Introduction}

Many daily activities that require both hands are intimately involved in functional and occupational activities, influencing the global, cognitive, emotional, and social motor development of a child. ${ }^{1}$ Complications in early childhood, such as cerebral palsy (CP) and brachial plexus injury (BPI), may cause asymmetry in the use of the upper limbs (UL) due to structural abnormalities, and an ample sensorimotor impairment, including muscle weakness, reduced bilateral coordination and impairments in postural control, in weight transfer, balance, locomotion, and the performance of daily activities. $^{2,3}$ Also, the least affected hemisphere is predominantly the individual's first choice in performing tasks and handling objects with, while the most affected limb can often be neglected. ${ }^{4}$ Thus, many hemiparetic children use only their unaffected limb to perform tasks usually performed bimanually. ${ }^{5}$ Therefore, to rehabilitate children and infants' asymmetry is crucial because, at this stage, there is an intense development of the nervous system that is influenced and modulated by the activities and experiences lived. ${ }^{6}$

The literature has currently shown two main techniques used to improve the manual or bimanual function of children with asymmetry, which have demonstrated positive results: The Constraint-Induced Movement Therapy (CIMT) and The Hand-Arm Bimanual Intensive Therapy (HABIT). While both therapies have a similar objective, the approach is different as CIMT focus mainly on unimanual dexterity, whereas HABIT focus on increasing the capacity of the bimanual performance. ${ }^{7-9}$

The CIMT presents a rehabilitation protocol for motor function and to overcome the learned nonuse, which influences daily activities and functional independence, ${ }^{10}$ and combines structured and systematized elements that restrict the non-affected side to encourage the use of the affected limb. ${ }^{11}$ Although CIMT can improve the quantity and quality of the affected upper limb movements, it does not focus on developing bimanual handling and coordination capacity as its objective, since the unaffected limb is restricted most of the time. Although studies have shown that unimanual practices can transfer improvements to bimanual coordination, ${ }^{10}$ motor learning principles emphasize the importance of task specificity in practice to maximize learning. ${ }^{12}$ Therefore, the direct training of bimanual skills corroborates to the improvement of bimanual coordination. ${ }^{6}$

In 2014, nine experts proposed a consensus to distinguish the CIMT from other training models for upper limbs. ${ }^{13}$ The CIMT is the original model, whose criteria involve the restraint of UL unaffected by $90 \%$ during waking hours, intensive training with the affected UL for at least 6 hours daily, and a minimum duration of 2 weeks. ${ }^{13}$ The modified restriction-induced motion therapy (mCIMT) involves the type of restriction (sling, 
cast, mitt/glove), the structured training (shaping/ repetition, motor learning), the intensity (hours per day) and duration (number of weeks) of the program, the location (home/camp), context (individual/group), and training provider (therapist/parents). ${ }^{13}$ The CIMT Hybrid models incorporate a bimanual component to the training (concomitant or immediately after the practice), maintaining the restriction and the intensity of training but adding the bimanual training to different extensions, changing the unimanual construction of the method significantly. ${ }^{13}$

Following the structure of CIMT Hybrid, the HABIT is an intensive therapy, which works on bimanual activities that use hands and arms and aims at the recovery or functional learning through the principles of motor learning and neuroplasticity. The HABIT does not restrict any of the limbs, providing structured and intensive bimanual practices with playing and functional activities. $^{5,9}$

Few studies focused on the CIMT and HABIT with infants, and none, up until now, presented the use of the combined approach in the stimulation of bimanual abilities in infants. Eliasson et al. ${ }^{13}$ pointed that among 24 randomized controlled trials and controlled clinical trials using the CIMT, 23 studies needed to alter at least one variable (a type of restriction, time to application, intensity, etc.) due to the age of the child. The adaptations performed, using the HABIT, were related to time and intensity of application due to the child. ${ }^{5}$

Concerning the duration and intensity of application, both methods originally propose the practice of 6 hours daily, from 10 to 21 days. ${ }^{9,11}$ However, it can be modified and adapted to become more suitable for younger children, restricting physical and psychological risks. ${ }^{5,6}$ In the original protocol of CIMT, the sessions' organization involves a set of behavioral methods that are applied to transfer the clinical situation's abilities to the real environment, recommending home-oriented tasks. ${ }^{11}$

Among the studies comparing the use of CIMT and HABIT in children with unilateral cerebral palsy, Sakzewski et al. ${ }^{8}$ indicated minimal differences between these two approaches since both present significant and similar improvements to the UL, however in older children. The present study evaluates the application of an intervention protocol based on the foundations of CIMT and HABIT to the improvement of the bimanual functionality of infants with UL asymmetry.

\section{Methods}

These are case studies of an applied nature, with a qualitative-quantitative approach and exploratorydescriptive content, which have followed all ethical research criteria (Certificate of Presentation for Ethical Consideration: n. 1.011.008). There is a focus on rehabilitating infants with asymmetry due to neurological disorder (central - diagnosed with CP or peripheral diagnosed with BPI).

The infants were recruited through an open invitation in private clinics and public pediatric and neonatology services in three cities in the state of São Paulo (Brazil). Were eligible for the study: (1) infants aged 6 to 24 months; (2) that feature asymmetry of the upper limbs due to non-evolutionary and non-degenerative neurological changes central (unilateral or bilateral CP) or peripheral (BPI). Were excluded from this study: (1) infants who had received botulinum toxin application within six months before the study start; (2) had previously participated in some constraint therapy protocol; (3) which did not understand simple verbal commands; (4) report of cognitive, auditory or visual impairment; (5) or did not present any degree of spontaneous range of movement (Figure 1). Although asymmetry is a clinical observation, we included only infants after the neurologist or pediatricians' diagnostic confirmation accompany them.

Five infants with UL's asymmetry participated in this study, three girls and two boys; four present $\mathrm{CP}$ diagnoses and one presented BPI. About the hemiparesis, three are spastic, one is spastic bilateral, and one is mild hypotonic. All infants were able to understand simple verbal commands (when the baby responds with motor action, whether pointing, looking to questions like "get the ball", "send kiss", "where's mommy?") to execute the activities performed during the intervention. The participant with BPI has Erb-Duchene's palsy, which is the mildest form of palsy with preservation of sensitivity and manual function; still, he did not use the upper limbs together, neglecting his affected UL. Thus, it allowed the subject to be included in the study, as it had asymmetry of UL and the other inclusion criteria.

Patients undergoing physiotherapy and occupational therapy maintained their usual treatment during the application of this protocol. Only patient P3 did not participate in any rehabilitation program since his injury was recent. Table 1 shows the characteristics of participants and their families. 


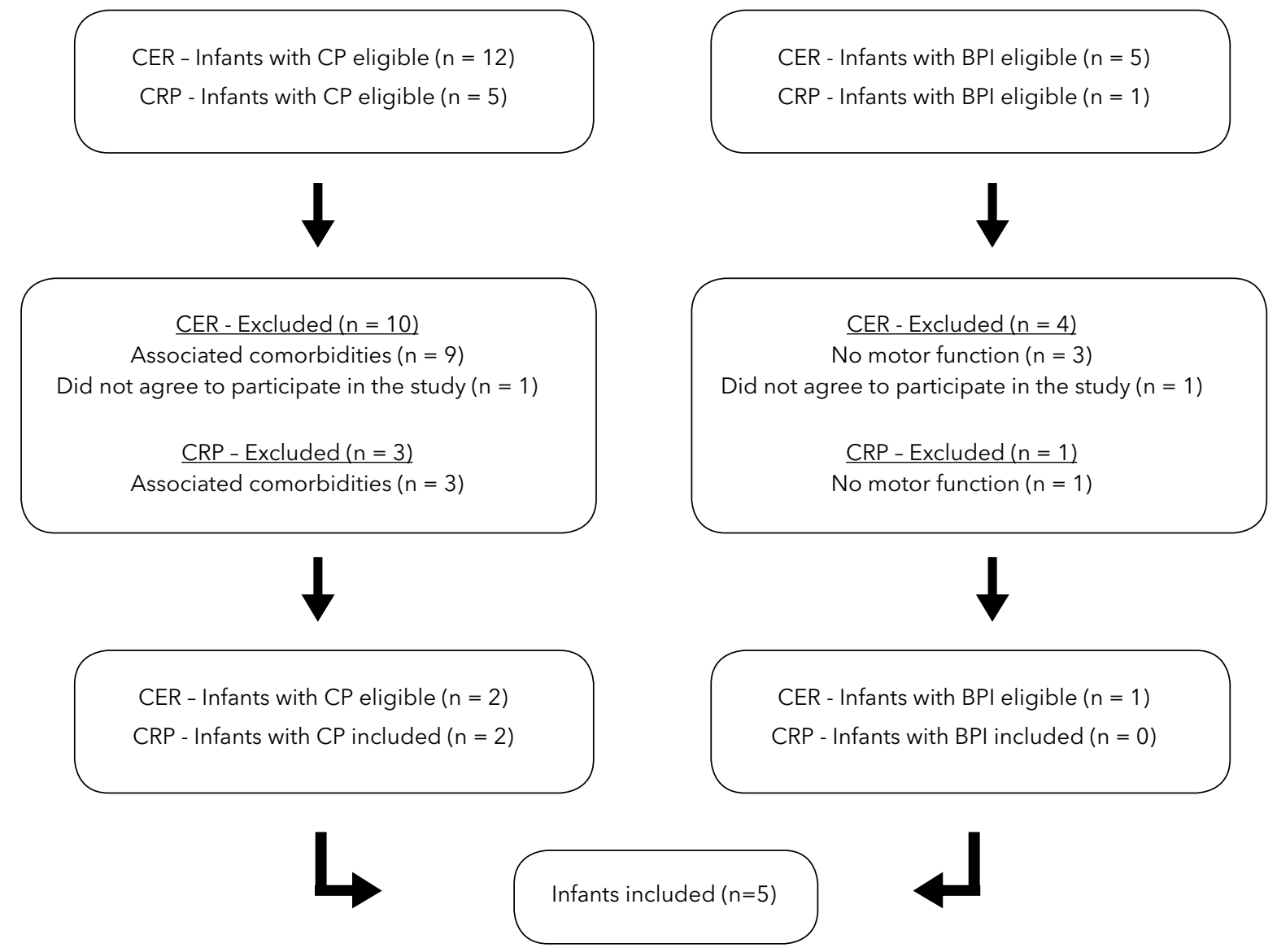

Figure 1 - Flowchart of inclusion and exclusion of patients from the CER (Rehabilitation Center of Ribeirão Preto) and CRP (Rehabilitation Center of Piracicaba) with cerebral palsy (CP) and brachial plexus injury (BPI).

Table 1 - Participants and families characterization

\begin{tabular}{|c|c|c|c|c|c|c|c|}
\hline Participants & Sex & $\begin{array}{l}\text { Chronological } \\
\text { age (months) }\end{array}$ & Diagnostic & Caregiver & Schooling & Age & $\begin{array}{c}\text { Monthly } \\
\text { family income } \\
\text { (U\$) }\end{array}$ \\
\hline P1 & M & 18 & $\begin{array}{l}\text { CP spastic hemiparetic } \\
\text { bilateral, GMFCS - IV; } \\
\text { Mini-MACS - IV }\end{array}$ & Grandmother & $\begin{array}{l}\text { Incomplete } \\
\text { Middle School }\end{array}$ & 41 & 519.16 \\
\hline P2 & $\mathrm{F}$ & 20 & $\begin{array}{l}\text { CP spastic hemiparetic, } \\
\text { GMFCS - I; Mini-MACS - II }\end{array}$ & Grandmother & High School & 38 & 1550.15 \\
\hline P3 & $\mathrm{F}$ & 20 & $\begin{array}{c}\text { CP spastic hemiparetic, } \\
\text { GMFCS - I; Mini-MACS - I }\end{array}$ & Mother & Middle School & 19 & 519.16 \\
\hline P4 & $\mathrm{F}$ & 6 & $\begin{array}{l}\text { CP spastic hemiparetic, } \\
\text { GMFCS - III; Mini-MACS - III }\end{array}$ & Mother & University & 24 & 1550.15 \\
\hline P5 & M & 15 & $\begin{array}{l}\text { Brachial Plexus Injury; } \\
\text { Erb-Duchene }\end{array}$ & Grandmother & $\begin{array}{c}\text { Elementary } \\
\text { School }\end{array}$ & 46 & 519.16 \\
\hline
\end{tabular}

Note: $\mathrm{M}=$ male; $\mathrm{F}=$ female. $\mathrm{CP}=$ Cerebral palsy; GMFCS = Gross Motor Function Classification System; Mini-MACS = Mini-Manual Ability Classification System. 


\section{Evaluation/ classification tolls}

Gross Motor Function Classification System for Cerebral Palsy Expanded and Revised (GMFCS E\&R): It has five classification levels according to the child's mobility, being level I more independent and level V total dependent, according to characteristics in each of five groups of age. ${ }^{14}$ The present study used the age group before the 2 nd birthday to classify infants' gross motor function with CP.

Mini-Manual Ability Classification System (MiniMACS): It is a hand ability classification system for children with CP aged from 1 to 4 years. ${ }^{15}$ Although the Mini-MACS is not adapted culturally to Brazilian children, we decided to use it because it is the most updated and appropriate version for the age investigated.

Characterization form of the participants: The researchers developed it to obtain information about infant development, pre-, peri and postnatal period, clinical data, maternal obstetric and family background, schooling of the primary caregiver, and the family's socioeconomic status.

Pediatric Evaluation of Disability Inventory (PEDI): It is a standardized questionnaire, appropriate for children aged 6 months to 7 years old, sensitive to small longitudinal changes, divided into three parts: Part I - assessing the child at tasks and daily activities in the areas of self-care, mobility and social function; Part II assessing the child's independence through the help provided by the caregiver, in the context of self-care, mobility, and social function; and Part III - if a child uses some modification in the environment to facilitate his/ her performance in functional tasks. ${ }^{16}$ In this research, we applied with the primary child's caregiver only Part I of the PEDI since our objective focuses on the individual's functional performance of the infant in their home environment.

Pediatric Motor Activity Log Revised (PMAL-R): Developed to measure the frequency the child uses his affected limb and spontaneous movement quality during functional activities. PMAL-R is a semi-structured interview administered to the child's primary caregiver of children from 2 to 8 years, created and standardized exclusively to evaluate the application of the induced restraint therapy method. ${ }^{17}$ Despite our participants being younger, we can observe many PMAL activities in infants, and remove/exclude from the calculation some items that do not match the child's chronological age. ${ }^{18}$
Therefore, we didn't apply high complexity motor tasks to children to not impairment in the evaluation.

Manual Function Evaluation (AMIGO): It measures the performance of the manual function of children from 0 to 7 years and 11 months, through the therapist's judgment. The abilities assessed in AMIGO follow an order of progressive complexity involving the areas of range, object manipulation, and grasping. ${ }^{19}$

\section{Procedures}

The intervention program used the modified restriction approach in stimulating bimanual skills based on the CIMT and HABIT theoretical foundations, and involved five consecutive weekly sessions (Monday to Friday), each session lasting 1 hour for four weeks. The details of this program are published in Correr and Pfeifer. ${ }^{20}$

The evaluations occurred on three moments: before the intervention program, shortly after, and four months of follow-up. We studied each child for a total of five months. The infants presented themselves comfortably (with the least possible clothes), and the interventions occur using toys and verbal guidance from the therapist in a previously organized room. ${ }^{20}$ Caregivers were present at all meetings (assessment and intervention) and actively participated in the therapy. They could do questions at any point in the process and received daily information about the importance of motor experiences and the child's bimanual exploration. The families received an allowance used for food and/or transportation. In the holidays, the therapist went to the patients' homes to avoid interruption of treatment.

Regarding the type of restriction, using a removable glove (made of neoprene and Velcro material) was chosen because it facilitates and allows the realization of bimanual activities. The activities were established according to each child's capacity, needs and interests, and it followed a progressive complexity pattern, which allowed the stimulation of motor development in bimanual activities. For further details on the description of the procedures and activities performed, see Correr and Pfeifer. ${ }^{20}$

\section{Statistical analysis}

Data were analyzed by the Jacobson-Truax (JT) method to verify the reliability of the changes between 
the pre- and post-intervention scores on the Reliable Change Index $(\mathrm{RCl})$ and the clinical significance of such changes. ${ }^{21}$ The $\mathrm{RCl}$ determines whether changes between assessments are due to the procedures used or are measurement errors. At the same time, clinical significance analysis verifies the degree to which patients have reached improvement or recovery patterns. $^{21}$

\section{Results}

We used the JT method to analyze the reliability of the changes between the pre- and post-intervention scores, the Reliable Change Index (RCl) (Table 2), and the clinical significance of the changes (Figure 2). The $\mathrm{RCl}$ analyzes the reliability of measurements and is reliable and positive when it exceeds 1.96 .

Table 2 - Participants and families characterization

\begin{tabular}{|c|c|c|c|c|c|}
\hline Participant & Evaluation & Pre-intervention & Post-intervention & Follow up & RCI (pre-, post-) \\
\hline \multirow{6}{*}{ P1 } & PEDI Self-care & 27.02 & 35.31 & 32.74 & 3.47 \\
\hline & PEDI Mobility & 6.97 & 11.38 & 14.24 & 2.17 \\
\hline & PEDI Social function & 34.24 & 37.01 & 40.60 & 2.50 \\
\hline & PMAL-R quantity & 5.00 & 12.00 & 27.00 & 0.55 \\
\hline & PMAL-R quality & 3.00 & 11.50 & 19.50 & 0.65 \\
\hline & AMIGO & 2.00 & 10.00 & 7.00 & 2.82 \\
\hline \multirow{6}{*}{ P2 } & PEDI Self-care & 49.34 & 55.05 & 55.76 & 2.39 \\
\hline & PEDI Mobility & 45.26 & 56.26 & 58.20 & 5.42 \\
\hline & PEDI Social function & 47.45 & 50.02 & 51.67 & 2.32 \\
\hline & PMAL-R quantity & 0.00 & 39.50 & 38.00 & 3.11 \\
\hline & PMAL-R quality & 0.00 & 48.50 & 23.00 & 3.73 \\
\hline & AMIGO & 30.00 & 38.00 & 38.00 & 2.11 \\
\hline \multirow{6}{*}{ P3 } & PEDI Self-care & 37.68 & 46.24 & 49.34 & 3.58 \\
\hline & PEDI Mobility & 36.80 & 46.67 & 51.94 & 4.86 \\
\hline & PEDI Social function & 47.45 & 48.32 & 50.02 & 0.78 \\
\hline & PMAL-R quantity & 9.00 & 74.50 & 81.00 & 5.15 \\
\hline & PMAL-R quality & 14.00 & 75.50 & 102.00 & 4.73 \\
\hline & AMIGO & 26.00 & 32.00 & 34.00 & 2.11 \\
\hline \multirow{6}{*}{ P4 } & PEDI Self-care & 35.31 & 39.85 & 52.94 & 1.90 \\
\hline & PEDI Mobility & 6.97 & 14.24 & 27.85 & 3.58 \\
\hline & PEDI Social function & 24.85 & 29.16 & 47.45 & 3.88 \\
\hline & PMAL-R quantity & 12.00 & 48.00 & 45.00 & 2.83 \\
\hline & PMAL-R quality & 20.50 & 49.50 & 48.00 & 2.23 \\
\hline & AMIGO & 20.00 & 24.00 & 26.00 & 1.41 \\
\hline \multirow{6}{*}{ P5 } & PEDI Self-care & 43.69 & 44.57 & 49.34 & 0.37 \\
\hline & PEDI Mobility & 48.11 & 52.76 & 51.94 & 2.29 \\
\hline & PEDI Social function & 46.56 & 47.45 & 49.18 & 0.80 \\
\hline & PMAL-R quantity & 44.50 & 77.50 & 74.00 & 2.59 \\
\hline & PMAL-R quality & 46.50 & 76.00 & 67.50 & 2.27 \\
\hline & AMIGO & 17.00 & 31.00 & 34.00 & 4.93 \\
\hline
\end{tabular}

Note: $\mathrm{RCI}=$ Reliable Change Index; PEDI = Pediatric Disability Assessment Inventory; PMAL-R = Pediatric Motor Activity Log; AMIGO = Manual Function Evaluation. 

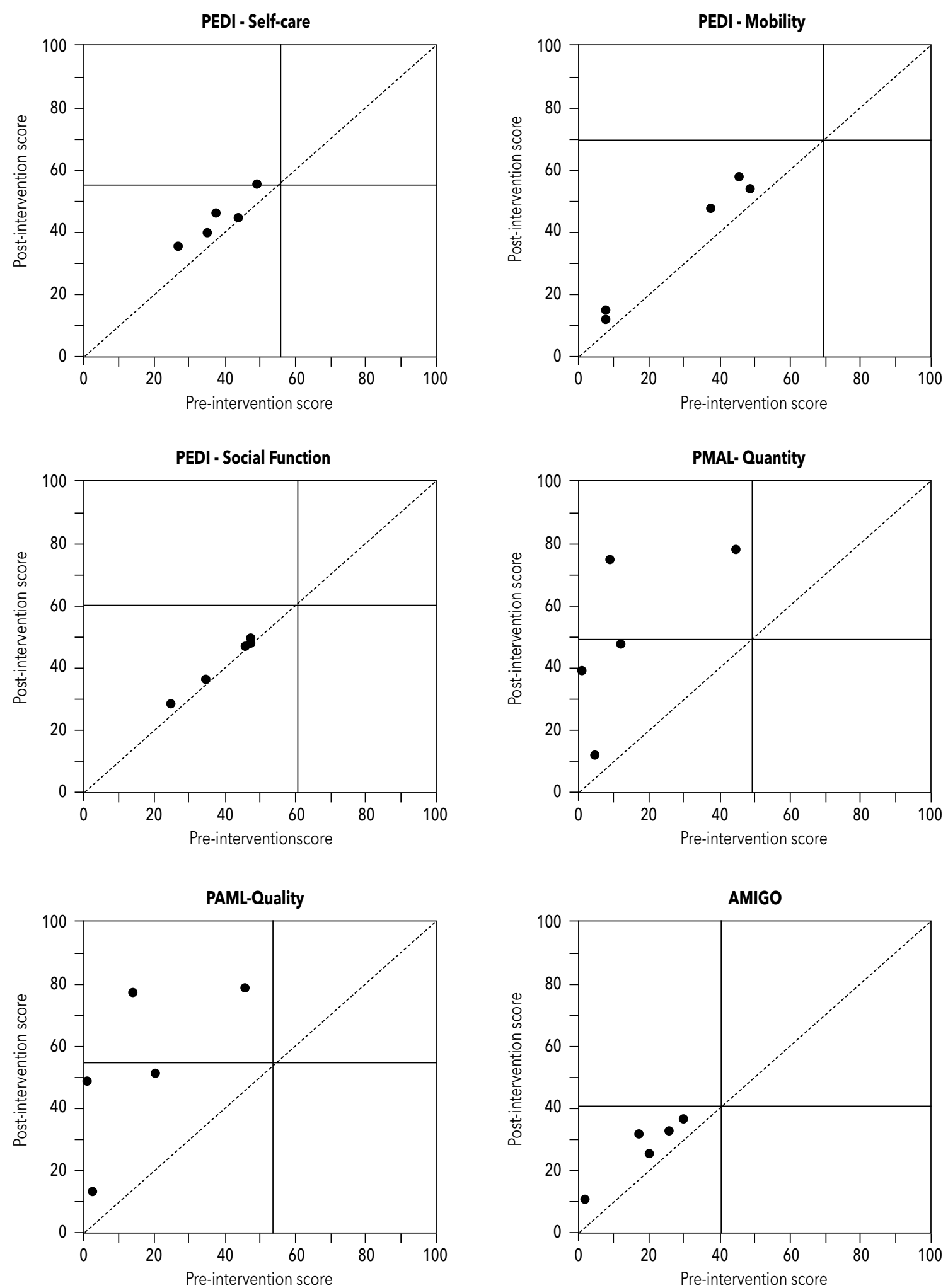

Figure 2 - Analysis of clinical significance the PEDI (Pediatric Disability Assessment Inventory), PMAL (Pediatric Motor Activity Log) and AMIGO (Manual Function Evaluation). 
The PEDI self-care session results presented an average of $38.6( \pm 8.4)$ in the evaluation and an average of $44.2( \pm 7.4)$ in the reevaluation. We can infer that P4 and P5 did not present reliable changes between the assessments. The clinical significance analysis showed that P1, P2, P3, and P4 reached the improvement proposed by the study, with P2 above the cutoff point (55.5 \pm 7.4$)$, which delimits the dysfunctional and functional zone. The infant who benefited the most from this rehabilitation protocol concerning self-care was P3, followed by P1.

The scores of PEDI - Mobility presented an average of $28.8( \pm 20.3)$ in the pre-intervention and $36.28( \pm 21.7)$ in the post-intervention. All infants showed an $\mathrm{RCl}$, ensuring that the procedures used did not constitute measurement errors. The analysis of clinical significance uses a cutoff point of 69.6 and a confidence interval of \pm 17.8 . In this analysis, we observed a high confidence interval because the group's standard deviation was high since they differed significantly regarding functional capacity, either by age or by the severity of the condition. However, all infants presented positive and favorable results with clinical significance. The infants who improved the most were P2 and P3, with differences of 11 and 10 points, respectively, in the continuous score. The P1 was the infant less benefited, with an $\mathrm{RCl}$ of 2.17 $(>1.96)$ and a difference of 4.4 in the continuous score.

The PEDI - Social Function showed an average of $40.1( \pm 10.2)$ in the pre-intervention and $42.3( \pm 8.9)$ in the post-intervention. Infants P3 and P5 did not present an $\mathrm{RCl}$. In the analysis of clinical significance, all infants are close to the bisector. The cutoff point for this analysis was 60.5 , and the confidence intervals were \pm 8.9 .

The PMAL-R - quantity and quality results evidenced a highly positive clinical significance in all infants. However, P1 did not present an RCl like the other infants since the affected limb's use was less than in the other participants. The standard deviation of the group was high in both evaluations. P1 presents spastic hemiparesis bilateral, and at the end of the protocol showed a more significant movement of the most affected UL; he modified his manual preference.

Infants P 3 and P5 changed into the functional quadrant of the dysfunctional population. The P3 caregiver reported his right-handed preference when picking up toys and food with functional movements. However, P3 had a stroke and was recruited shortly after discharge, ensuring early intervention. At the beginning of the treatment, P3 neglected the affected UL (formerly used to play), but, at the end of treatment, the right-hand motor sequelae were hardly noticeable in the performance of tasks; however, the child still preferred the left hand in his activities.

The infant P5 was diagnosed with a brachial obstetric injury, with manual function preserved. By improving the amount of hand use, the infant moved to the functional quadrant. However, tasks involving reaching and gripping above the nipple line are still tricky and performed without high shoulder flexion degrees.

Regarding the manual function, assessed by AMIGO, all infants achieved an $\mathrm{RCl}$. P5 presented the most significant changes in the performance since their capacity to perform the instrument's functional activities had not been affected by the peripheral lesion. The first score of P5 in AMIGO was low because he had no experience in the proposed activities. Lack of stimulation, associated with limited motion, was the principal cause of their delayed motor skills. Infant P1 also presented a significant change, going from 2 points in the preevaluation to 10 points in the post-intervention period.

It is noteworthy that the therapy was applied in only weeks, and the performance achieved was recorded. At the end of these 4 weeks, participants continued their usual therapies for 16 weeks without receiving CIMT or HABIT and were then reassessed (follow-up). In this evaluation, we observed the acquisitions that were maintained, as well as the new performances. However, what was achieved after 16 weeks was as good as or less than the gains achieved in the 4 weeks of intensive intervention proposed here (see Table 2).

\section{Discussion}

Of all disorders caused by hemiparesis, manual function alteration is the most disabling since many children perform typically bimanual activities, using only the unaffected UL. ${ }^{5}$ The present study recruited infants aged 6 to 24 months with hemiparesis, focused on applying an effective rehabilitation method to the upper limbs. Only three hemiparesis $C P$, one bilateral hemiparesis, and one BPI participated in this study. We decided not to include participants who did not present a complete $\mathrm{CP}$ diagnosis, avoiding bias if the asymmetry was due to only a delay in development. It is a challenge to perform an early intervention with babies with CP since this diagnosis occurs at around 12 to 24 months of age, ${ }^{22}$ unilateral lesions do not necessarily 
lead to unilateral $\mathrm{CP}$, and the children that are suffering from neonatal stroke, only 30\% develop unilateral $\mathrm{CP}^{23}$ In addition to the difficulties with early diagnosis, the diagnostic subtype may change the first years of life. ${ }^{24}$ For patients with BPI to be included, there was the need to have some hand function; many who were in rehabilitation services presented hand function with severe consequences, or they were older and, therefore, did not fit in the inclusion criteria.

It wasn't easy to perform research with this population. A critical study published by Eliasson et al. ${ }^{25}$ evaluated the effectiveness of Baby-CIMT in infants younger than 12 months. This study demanded six years, and the recruitment of infants was into several followup programs. Were eligible infants aged between 3 and 8 months of corrected age and high risk of developing unilateral CP. The risks were identified by a neurologist or physiotherapist using assessments such as the Alberta Infant Motor Scale (AIMS) or Hammersmith Infant Neurological Examination (HINE); ${ }^{7}$ also, they needed to have a $\geq 15 \%$ difference between the two hands evaluated by Hand Assessment for Infants (HAI). ${ }^{26}$ They selected thirty-seven participants but excluded six because they did not confirm the CP diagnoses at the end of the study.

Early diagnosis allows timely access to early intervention when the most significant gains from neuroplasticity are possible. On the other hand, late diagnosis can be detrimental to a child's development. ${ }^{22}$ The CIMT is applied widely in individuals with unilateral $\mathrm{CP}$ and other forms of $\mathrm{CP}$ or pathologies with asymmetric functional deficits of upper limbs. ${ }^{27}$ As the study recruited participants at an early age, it was necessary to adapt the CIMT protocol, increase the participants' tolerance and adherence, and associate it with the HABIT protocol. Our study differs from the other hybrid models that opted to work bimanual activities in the last days of CIMT treatment ${ }^{28}$ or immediately after. ${ }^{29}$ We chose to organize the intervention program with tasks previously selected to perform using two hands together. ${ }^{20}$ The less-affected arm was slightly restricted by a glove, allowing less selective movements. ${ }^{20}$

To arm restriction, we used a removable glove made of neoprene, which extends up to the middle of the forearm, ${ }^{20}$ similar to the study by Coker et al. ${ }^{30}$ This option does not exclude the containment character of the CIMT, as it inhibits the exclusive use of the unaffected ${ }^{30}$ UL and allows the application of HABIT. The removable glove prevents the infant from using the unaffected UL's fine motor skills, although it can be used as support in bimanual activities. Just as importantly, the glove allows freedom of movement of the shoulder, elbow and wrist, allowing transfer tasks, laterality, and protection from falls. ${ }^{9}$ The infants showed no irritability when using the glove and showed no signs of stress from restriction.

Interventions using CIMT in children differ widely regarding the intensity of the sessions and the time spent with the contention device; these can range from sessions of 2 hours $^{10}$ up to 6 hours daily, ${ }^{31}$ and limb restraint between 2 hours ${ }^{10}$ to 24 hours per day. ${ }^{30}$ Since our work is with infants, we proposed a protocol of 5 weekly sessions, lasting 1 hour per day, for four weeks.

Our study adopted behavioral methods by including the caregivers in the sessions and using motivational rewards for the infants, predominantly verbal praise, smiles, hugs, and clapping. We explained to the caregivers the importance of each activity's in the infant's manual function and discussed all questions regarding the condition, prognosis, and abilities. We instructed the caregivers to encourage the infants at home and not criticize them if they presented difficulty or failure in a particular function or activity. We did not directed the home-oriented tasks to avoid bias, in which one family could participate more or less than the other. ${ }^{20}$ Three of the patients lived with their grandmothers and two with their parents; the caregivers presented distinct levels of formal education, financial resources, and time spent with their children.

The infants maintained their rehabilitation routines in physical therapy and occupational therapy throughout the present study's intervention. In the case of conventional rehabilitation being interrupted during the intensive intervention and returning immediately after its end, it could compromise the results, generating bias regarding what was or was not maintained. With the manutention of a conventional rehabilitation routine, it is possible to measure the speed and intensity of the gains before and after the intensive intervention proposed here. Also, infants are not allowed to remain without intervention during the follow-up period.

The results presented here are similar to those of Cope et al., ${ }^{32}$ that also used a modified protocol of induced restraint therapy and demonstrated a significant improvement in the movement and function of UL children with hemiparesis. Also are similar to those reported by Gordon et al., ${ }^{9}$ who implemented a HABIT 
protocol of 6 hours daily, for 10 days, in children with spastic hemiparesis, indicating efficacy and increase of the bimanual function of the experimental group. However, our study differs in that it is more efficient in younger children, with a daily intensity of only 1 hour and less invasive restriction. Similar to ours, results were also obtained by Eliasson et al., ${ }^{10}$ who used a more modified CIMT protocol, whose intervention was applied by parents or teachers and supervised weekly by a professional, and demonstrated an improvement of hemiparetic hand utilization capacity.

\section{Study limitations}

The main limitations are the sample's size and heterogeneity, not allowing the formation of a control group. Therefore, we used the JT Method, a procedure utterly distinct from inferential statistics since it is possible to find reliable changes without statistically significant differences. Although without a return to normal functioning, a clinically significant change can make a big difference in the life of the individual, especially in cases with sensory or motor disorders. ${ }^{33}$ This method is an alternative for studies with a small number of subjects, a lack of normative and control group data that makes an inferential analysis of the results impossible based on central tendency and dispersion. ${ }^{21}$ Another aspect is the absence of standardized and appropriate instruments for infants ( $<2$ years) to evaluate the unilateral function; therefore, PMAL is frequently used in CIMT studies with infants. ${ }^{18,30,32}$ Eliasson et al. ${ }^{25}$ used the new HAl, ${ }^{26}$ a recently developed evaluation measure to estimate the performance of the upper extremities of children younger than 12 months at high risk of unilateral cerebral palsy. As far as we know, however, this instrument was not validated for the Brazilian population during our research.

\section{Conclusion}

This study allowed us to observe an increase in the uni and bimanual function in infants, hypothesizing that treatment at an early age positively influences sensorymotor development in infants with nervous system injuries since there are presumably critical periods during the maturation of the developing brain that ensure greater effectiveness at earlier ages.

\section{Acknowledgments}

We would like to thank the infants and their families for their participation. As well, we would like to thank the Coordenação de Aperfeiçoamento de Pessoal de Nivel Superior (CAPES) for the master and doctoral scholarship to the first author, and to Conselho Nacional de Desenvolvimento Científico e Tecnológico (CNPQ) for the research productivity scholarship to the second author.

\section{Authors' contributions}

MTCR was responsible for the investigation, formal analysis, writing-original draft and writing-review and editing. LIP was responsible for the the funding acquisition, methodology, project administration and supervision. Both authors were equally responsible for the conceptualization and data curation.

\section{References}

1. Chien CW, Brown T, McDonald R. A framework of children's hand skills for assessment and intervention. Child Care Health Dev. 2009;35(6):873-84. DOI

2. Ferrigno ISV. Terapia da mão: fundamentos para a prática clínica. São Paulo: Santos; 2007. 157 p.

3. Robert MT, Guberek R, Sveistrup H, Levin MF. Motor learning in children with hemiplegic cerebral palsy and the role of sensation in short-term motor training of goal-directed reaching. Dev Med Child Neurol. 2013;55(12):1121-8. DOI

4. Sköld A, Josephsson S, Eliasson AC. Performing bimanual activities: the experiences of young persons with hemiplegic cerebral palsy. Am J Occup Ther. 2004;58(4):416-25. DOI

5. Charles J, Gordon AM. Development of hand-arm bimanual intensive training (HABIT) for improving bimanual coordination in children with hemiplegic cerebral palsy. Dev Med Child Neurol. 2006;48(11):931-6. DOI

6. Martin JH, Friel KM, Salimi I, Chakrabarty S. Activity- and usedependent plasticity of the developing corticospinal system. Neurosci Biobehav Rev. 2007;31(8):1125-35. DOI 
7. Novak I, Morgan C, Adde L, Blackman J, Boyd RN, BrunstromHernandez J, et al. Early, accurate diagnosis and early intervention in cerebral palsy. JAMA Pediatr. 2017;171(9):897907. DOI

8. Sakzewski L, Ziviani J, Boyd RN. Efficacy of upper limb therapies for unilateral cerebral palsy: a meta-analysis. Pediatrics. 2014;133(1):e175-204. DOI

9. Gordon AM, Schneider JA, Chinnan A, Charles JR. Efficacy of a hand-arm bimanual intensive therapy (HABIT) in children with hemiplegic cerebral palsy: a randomized control trial. Dev Med Child Neurol. 2007;49(11):830-8. DOI

10. Eliasson AC, Krumlinde-Sundholm L, Shaw K, Wang C. Effects of constraint-induced movement therapy in young children with hemiplegic cerebral palsy: an adapted model. Dev Med Child Neurol. 2005;47(4):266-75. DOI

11. Taub E. The behavior-analytic origins of constraintinduced movement therapy: an example of behavioral neurorehabilitation. Behav Anal. 2012;35(2):155-78. DOI

12. Kitago T, Krakauer JW. Motor learning principles for neurorehabilitation. Handb Clin Neurol. 2013;110:93-103. DO

13. Eliasson AC, Krumlinde-Sundholm L, Gordon AM, Feys $\mathrm{H}$, Klingels $\mathrm{K}$, Aarts PBM, et al. Guidelines for future research in constraint-induced movement therapy for children with unilateral cerebral palsy: an expert consensus. Dev Med Child Neurol. 2014;56(2):125-37. DOI

14. Silva DBR, Pfeifer LI, Funayama CAR. Gross Motor Function Classification System Expanded \& Revised (GMFCS E \& R): reliability between therapists and parents in Brazil. Braz J Phys Ther. 2013;17(5):458-63. DOI

15. Eliasson AC, Ullenhag A, Wahlström U, Krumlinde-Sundholm L. Mini-MACS: development of the Manual Ability Classification System for children younger than 4 years of age with signs of cerebral palsy. Dev Med Child Neurol. 2017;59(1):72-8. DOI

16. Mancini MC. Inventário de avaliação pediátrica de incapacidade (PEDI): manual da versão brasileira adaptada. Belo Horizonte: UFMG; 2005. 193 p.

17. Uswatte G, Taub E, Griffin A, Vogtle L, Rowe J, Barman J. The pediatric motor activity log-revised: assessing real-world arm use in children with cerebral palsy. Rehabil Psychol. 2012;57(2):149-58. DOI

18. Matuti G, Santos J, Silva A, Eras-Garcia R, Uswatte G, Taub E. Translation and cross cultural adaptation of the Pediatric Motor Activity Log-Revised Scale. Arq Neuropsiquiatr. 2016;74(7):555-60. DOI

19. Sabino LAAS. Elaboração de um protocolo de avaliação da função manual de crianças com paralisia cerebral: etapa inicial [master's thesis]. Ribeirão Preto: Universidade de São Paulo; 2016. Full text link

20. Correr MT, Pfeifer LI. Intensive care protocol for infants with asymmetry. Cad Bras Ter Ocup. 2019;27(2):317-30. DOI

21. Del Prette ZAP, Del Prette A. Significância clínica e mudança confiável na avaliação de intervenções psicológicas. Psic Teor Pesq. 2008;24(4):497-505. DOI

22. McIntyre S, Morgan C, Walker K, Novak I. Cerebral palsydon't delay. Dev Disabil Res Rev. 2011;17(2):114-29. DOI

23. Husson B, Hertz-Pannier L, Renaud C, Allard D, Presles

$\mathrm{E}$, Landrieu $\mathrm{P}$, et al. Motor outcomes after neonatal arterial ischemic stroke related to early mri data in a prospective study. Pediatrics. 2010;126(4):912-8. DOI

24. Morgan C, Novak I, Dale RC, Guzzetta A, Badawi N. Single blind randomised controlled trial of GAME (Goals - Activity Motor Enrichment) in infants at high risk of cerebral palsy. Res Dev Disabil. 2016;55:256-67. DOI

25. Eliasson AC, Nordstrand L, Ek L, Lennartsson F, Sjöstrand $L$, Tedroff $K$, et al. The effectiveness of Baby-CIMT in infants younger than 12 months with clinical signs of unilateral-cerebral palsy; an explorative study with randomized design. Res Dev Disabil. 2018;72:191-201. DOI

26. Krumlinde-Sundholm L, Ek L, Sicola E, Sjöstrand L, Guzzetta A, Sgandurra $G$, et al. Development of the hand assessment for infants: evidence of internal scale validity. Dev Med Child Neurol. 2017:59(12):1276-83. DOI

27. DeLuca SC, Trucks MR, Wallace DA, Ramey SL. Practicebased evidence from a clinical cohort that received pediatric constraint- induced movement therapy. J Pediatr Rehabil Med. 2017;10(1):37-46. DOI 
28. Taub E, Griffin A, Uswatte G, Gammons K, Nick J, Law CR. Treatment of congenital hemiparesis with pediatric constraintinduced movement therapy. J Child Neurol. 2011; 26(9):1163-73. DOI

29. Brandão MB, Mancini MC, Vaz DV, Melo APP, Fonseca ST. Adapted version of constraint-induced movement therapy promotes functioning in children with cerebral palsy: a randomized controlled trial. Clin Rehabil. 2010;24(7):639-47. DOI

30. Coker P, Lebkicher C, Harris L, Snape J. The effects of constraint-induced movement therapy for a child less than one year of age. NeuroRehabilitation. 2009;24(3):199-208. DOI
31. DeLuca S, Echols K, Ramey SL, Taub E. Pediatric ConstraintInduced Movement Therapy for a young child with cerebral palsy: two episodes of care. Phys Ther. 2003;83(11):1003-13. $\mathrm{DOI}$

32. Cope SM, Forst HC, Bibis D, Liu XC. Modified constraintinduced movement therapy for a 12-month-old child with hemiplegia: a case report. Am J Occup Ther. 2008;62(4):430-7. $\mathrm{DOI}$

33. Evans C, Margison F, Barkham M. The contribution of reliable and clinically significant change methods to evidence-based mental health. Evid Based Ment Health. 1998;1(3):70-2. DOI 